\title{
Mécanique quantique tome 2
}





\section{Albert Messiah}

\section{Mécanique quantique fome 2}

\section{Préfaces de}

\section{Roger Balian}

Directeur de Recherches au CEA Professeur à l'École Polytechnique et Claude Cohen-Tannoudji Professeur au Collège de France

et de

Pierre-Gilles de Gennes

Professeur au Collège de France

Nouvelle Édition

DUNOD 
Ce pictogramme mérite une explicotion. Son objet est d'alerter le lecteur sur la menace que représente pour l'avenir de l'écrit, particulièrement dans le domaine de l'édition lechnique ef universitaire, le développement massif du photocopillage.

Le Code de la propriélé intellectuelle du ler juillet 1992 interdit en effet expressément la photocopie ò usage collectif sans autorisation des ayants droit. Or, cette pratique s'est généralisée dans les établisse- ments d'enseignement supérieur, provo quant une baisse brutale des achats de livres ef de revues, au point que la possibilité même pour les auteurs de créer des ceuvres nouvelles et de les faire éditer correctement est aujourd'hui menacée.

Nous rappelons donc que toute reproduction, partielle ov totale, de la présente publication est interdite sans autorisa-
Centre fronçais d'exploitation du troit de copie (CFC, 3 rue Hautefeuille, 75006 Paris).

(C) Dunod, Paris, 1995

ISBN 2 10002427-2

Toute représentation ou reproduction, intégrale ou partielle, faite sans le consentement de l'auteur, ou de ses ayants droit, ou ayants cause, est illicite (loi du 11 mars 1957, alinéa ler de l'article 40). Cette représentation ou reproduction, par quelque procédé que ce soit, constituerait une contrefaçon sanctionnée par les articles 425 et suivants du Code pénal. La loi du 11 mars 1957 n'autorise, aux termes des alinéas 2 et 3 de l'article 41, que les copies ou reproductions strictement réservées à l'usage privé du copiste et non destinées à une utilisation collective d'une part, et d'autre part, que les analyses les courtes citations dans un but d'exemple et d'illustration. 